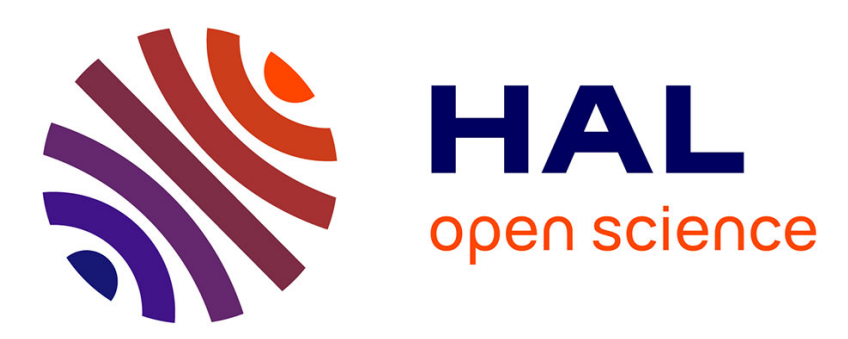

\title{
On some Gaussian Bernstein processes in RN and the periodic Ornstein-Uhlenbeck process
}

\author{
Pierre-A Vuillermot, Jean-C Zambrini
}

\section{To cite this version:}

Pierre-A Vuillermot, Jean-C Zambrini. On some Gaussian Bernstein processes in RN and the periodic Ornstein-Uhlenbeck process. Stochastic Analysis and Applications, 2016, 34 (4), pp.573-597. 10.1080/07362994.2016.1156547 . hal-01187015

\section{HAL Id: hal-01187015 \\ https://hal.science/hal-01187015}

Submitted on 25 Aug 2015

HAL is a multi-disciplinary open access archive for the deposit and dissemination of scientific research documents, whether they are published or not. The documents may come from teaching and research institutions in France or abroad, or from public or private research centers.
L'archive ouverte pluridisciplinaire HAL, est destinée au dépôt et à la diffusion de documents scientifiques de niveau recherche, publiés ou non, émanant des établissements d'enseignement et de recherche français ou étrangers, des laboratoires publics ou privés. 


\title{
On some Gaussian Bernstein processes in $\mathbb{R}^{N}$ and the periodic Ornstein-Uhlenbeck process
}

\author{
Pierre-A. Vuillermot* and Jean-C. Zambrini** \\ UMR-CNRS 7502, Inst. Élie Cartan de Lorraine, Nancy, France* \\ Departamento de Matemática, Universidade de Lisboa, Portugal ${ }^{* *}$
}

\begin{abstract}
In this article we prove new results regarding the existence of Bernstein processes associated with the Cauchy problem of certain forwardbackward systems of decoupled linear deterministic parabolic equations defined in Euclidean space of arbitrary dimension $N \in \mathbb{N}^{+}$, whose initial and final conditions are positive measures. We concentrate primarily on the case where the elliptic part of the parabolic operator is related to the Hamiltonian of an isotropic system of quantum harmonic oscillators. In this situation there are many Gaussian processes of interest whose existence follows from our analysis, including $N$-dimensional stationary and non-stationary Ornstein-Uhlenbeck processes, as well as a Bernstein bridge which may be interpreted as a Markovian loop in a particular case. We also introduce a new class of stationary nonMarkovian processes which we eventually relate to the $N$-dimensional periodic Ornstein-Uhlenbeck process, and which is generated by a oneparameter family of non-Markovian probability measures. In this case our construction requires the consideration of an infinite hierarchy of pairs of forward-backward heat equations associated with the pure point spectrum of the elliptic part, rather than just one pair in the Markovian case. We finally stress the potential relevance of these new processes to statistical mechanics, the random evolution of loops and general pattern theory.
\end{abstract}

\section{Introduction and outline}

Let us consider the two adjoint parabolic Cauchy problems

$$
\begin{aligned}
\partial_{t} u(\mathrm{x}, t) & =\frac{1}{2} \Delta_{\mathrm{x}} u(\mathrm{x}, t)-V(\mathrm{x}) u(\mathrm{x}, t), \quad(\mathrm{x}, t) \in \mathbb{R}^{N} \times(0, T], \\
u(\mathrm{x}, 0) & =\varphi_{0}(\mathrm{x}), \quad \mathrm{x} \in \mathbb{R}^{N}
\end{aligned}
$$

and

$$
\begin{aligned}
-\partial_{t} v(\mathrm{x}, t) & =\frac{1}{2} \Delta_{\mathrm{x}} v(\mathrm{x}, t)-V(\mathrm{x}) v(\mathrm{x}, t), \quad(\mathrm{x}, t) \in \mathbb{R}^{N} \times[0, T), \\
v(\mathrm{x}, T) & =\psi_{T}(\mathrm{x}), \quad \mathrm{x} \in \mathbb{R}^{N}
\end{aligned}
$$


where $T \in(0,+\infty)$ is arbitrary. In these equations, $\Delta_{\times}$denotes Laplace's operator with respect to the spatial variable, $V$ is a real-valued function while $\varphi_{0}$ and $\psi_{T}$ are positive measures on $\mathbb{R}^{N}$. Both (1) and (2) can then be looked upon as defining a forward-backward system of decoupled linear deterministic heat equations in Euclidean space. To wit, the potential solutions to (1) wander off to the future, whereas those of (2) evolve into the past. In Section 2 below we show that under very general conditions on $V, \varphi_{0}$ and $\psi_{T}$, we can associate with (1)(2) a class of the so-called Bernstein or reciprocal processes, henceforth denoted by $Z_{\tau \in[0, T]}$. These are processes that constitute a generalization of Markov processes which have played an increasingly important rôle in various areas of mathematics and mathematical physics over the years (see, e.g., [1], [3]-[5], [10], [18], [21] and the many references therein for a history and ealier works on the subject). In the case of (1)-(2) the state space of the processes is the entire Euclidean space, and their construction requires a transition function as well as a joint probability distribution for $Z_{0}$ and $Z_{T}$, which we denote respectively by $P$ and $\mu$ in the sequel. While $P$ depends exclusively on the parabolic Green function associated with (1)-(2), the measure $\mu$ involves both Green's function and $\varphi_{0}, \psi_{T}$, or more generally a statistical mixture of $\varphi_{0}$ and $\psi_{T}$. It is $P$ and $\mu$ that allow one eventually to write out all the finite-dimensional distributions of $Z_{\tau \in[0, T]}$, on which the remaining part of this article is based. In Section 3 we concentrate primarily on certain Markovian Gaussian Bernstein processes associated with a particular case of (1)-(2), namely,

$$
\begin{aligned}
\partial_{t} u(\mathrm{x}, t) & =\frac{1}{2} \Delta_{\mathrm{x}} u(\mathrm{x}, t)-\frac{\lambda^{2}}{2}|\mathrm{x}|^{2} u(\mathrm{x}, t), \quad(\mathrm{x}, t) \in \mathbb{R}^{N} \times(0, T], \\
u(\mathrm{x}, 0) & =\varphi_{0}(\mathrm{x}), \quad \mathrm{x} \in \mathbb{R}^{N}
\end{aligned}
$$

and

$$
\begin{aligned}
-\partial_{t} v(\mathrm{x}, t) & =\frac{1}{2} \Delta_{\mathrm{x}} v(\mathrm{x}, t)-\frac{\lambda^{2}}{2}|\mathrm{x}|^{2} v(\mathrm{x}, t), \quad(\mathrm{x}, t) \in \mathbb{R}^{N} \times[0, T), \\
v(\mathrm{x}, T) & =\psi_{T}(\mathrm{x}), \quad \mathrm{x} \in \mathbb{R}^{N}
\end{aligned}
$$

where $\lambda>0$ and |.| denotes the Euclidean norm in $\mathbb{R}^{N}$, whose right-hand side is the Hamiltonian of an isotropic system of quantum harmonic oscillators, up to a sign (see, e.g., [15]). There we apply the results of Section 2 in order to construct and analyze three processes of interest, by determining explicitly in each case the finite-dimensional projections of the corresponding Gaussian measures. The first one is stationary and thereby esssentially a $N$-dimensional Ornstein-Uhlenbeck process, while the second one is a non-stationary OrnsteinUhlenbeck process conditioned to start at the origin of $\mathbb{R}^{N}$. The third one is a process that shares many of the properties of a bridge, which we call a Bernstein bridge and which we may identify with a Markovian loop in a particular case. The measures $\mu$ we need for the construction of each one of these are intimately tied up with a very specific choice of initial-final data in (3)-(4). The situation is quite different in Section 4, where we construct a new family of stationary nonMarkovian Bernstein processes that are related to the $N$-dimensional periodic 
Ornstein-Uhlenbeck process. There, we prove that the relevant non-Markovian probability measures $\mu$ disintegrate into statistical mixtures of the form

$$
\mu=\sum_{\mathrm{m} \in \mathbb{N}^{N}} p_{\mathrm{m}} \mu_{\mathrm{m}}, \quad p_{\mathrm{m}}>0, \quad \sum_{\mathbf{m} \in \mathbb{N}^{N}} p_{\mathrm{m}}=1,
$$

where each $\mu_{\mathrm{m}}$ is a measure related to initial-final conditions $\varphi_{\mathrm{m}, 0}$ and $\psi_{\mathrm{m}, T}$ in

$$
\begin{aligned}
\partial_{t} u(\mathrm{x}, t) & =\frac{1}{2} \Delta_{\mathrm{x}} u(\mathrm{x}, t)-\frac{\lambda^{2}}{2}|\mathrm{x}|^{2} u(\mathrm{x}, t), \quad(\mathrm{x}, t) \in \mathbb{R}^{N} \times(0, T], \\
u(\mathrm{x}, 0) & =\varphi_{\mathrm{m}, 0}(\mathrm{x}), \quad \mathrm{x} \in \mathbb{R}^{N}
\end{aligned}
$$

and

$$
\begin{aligned}
-\partial_{t} v(\mathrm{x}, t) & =\frac{1}{2} \Delta_{\mathrm{x}} v(\mathrm{x}, t)-\frac{\lambda^{2}}{2}|\mathrm{x}|^{2} v(\mathrm{x}, t), \quad(\mathrm{x}, t) \in \mathbb{R}^{N} \times[0, T), \\
v(\mathrm{x}, T) & =\psi_{\mathrm{m}, T}(\mathrm{x}), \quad \mathrm{x} \in \mathbb{R}^{N},
\end{aligned}
$$

respectively. In other words, we show there that the construction of the $\mu$ 's given by (5) requires the consideration of a hierarchy of infinitely many pairs of problems of the form (3)-(4) associated with the whole pure point spectrum of the elliptic operator on the right-hand side, rather than just one pair in the Markovian case. Finally, we also point out the potential applications of those new processes to statistical mechanics, to the problem of random evolution of loops in space and to general pattern theory, to name only three. In an appendix we also prove an important series expansion for the Green function associated with(3)-(4).

\section{A class of Bernstein processes in $\mathbb{R}^{N}$}

Generally speaking Bernstein processes can take values in any topological space countable at infinity, and there are several equivalent ways to characterize them (see, e.g., [10]). However, the following definition will be sufficient for our purposes:

Definition 1. Let $N \in \mathbb{N}^{+}$and $T \in(0,+\infty)$ be arbitrary. We say the $\mathbb{R}^{N}$-valued process $Z_{\tau \in[0, T]}$ defined on the complete probability space $(\Omega, \mathcal{F}, \mathbb{P})$ is a Bernstein process if

$$
\mathbb{E}\left(f\left(Z_{r}\right) \mid \mathcal{F}_{s}^{+} \vee \mathcal{F}_{t}^{-}\right)=\mathbb{E}\left(f\left(Z_{r}\right) \mid Z_{s}, Z_{t}\right)
$$

for every bounded Borel measurable function $f: \mathbb{R}^{N} \mapsto \mathbb{R}$, and for all $r, s, t$ satisfying $r \in(s, t) \subset[0, T]$. In (8), the $\sigma$-algebras are

$$
\mathcal{F}_{s}^{+}=\sigma\left\{Z_{\tau}^{-1}(E): \tau \leq s, E \in \mathcal{B}_{N}\right\}
$$

and

$$
\mathcal{F}_{t}^{-}=\sigma\left\{Z_{\tau}^{-1}(E): \tau \geq t, E \in \mathcal{B}_{N}\right\}
$$


where $\mathcal{B}_{N}$ stands for the Borel $\sigma$-algebra on $\mathbb{R}^{N}$.

The dynamics of such a process at any time $r \in(s, t)$ are, therefore, solely determined by the properties of the process at times $s$ and $t$, irrespective of its behavior prior to instant $s$ and after instant $t$. Of course, it is this fact that generalizes the Markov property.

In order to associate a mere Bernstein process with (1)-(2) we now impose the following hypothesis, which regards Green's function alone:

(H1) The measurable function $V: \mathbb{R}^{N} \mapsto \mathbb{R}$ is such that the parabolic Green function $g$ associated with (1)-(2) is jointly continuous in all variables and satisfies

$$
g(\mathrm{x}, t, \mathrm{y})>0
$$

for all $\mathrm{x}, \mathrm{y} \in \mathbb{R}^{N}$ and every $t \in(0, T]$.

Let us now write $\mathcal{M}\left(\mathbb{R}^{N} \times \mathbb{R}^{N}, \mathbb{C}\right)$ for the space of measures we are interested in, namely, the topological dual of the Fréchet space of all complex-valued, compactly supported functions on $\mathbb{R}^{N} \times \mathbb{R}^{N}$ endowed with the usual locally convex topology (see, e.g., [19]). Having (11) at our disposal, let us introduce the functions

$$
p(\mathrm{x}, t ; \mathrm{z}, r ; \mathrm{y}, s):=\frac{g(\mathrm{x}, t-r, \mathrm{z}) g(\mathrm{z}, r-s, \mathrm{y})}{g(\mathrm{x}, t-s, \mathrm{y})}
$$

and

$$
P(\mathrm{x}, t ; E, r ; \mathrm{y}, s):=\int_{E} d \mathrm{z} p(\mathrm{x}, t ; \mathrm{z}, r ; \mathrm{y}, s)
$$

for every $E \in \mathcal{B}_{N}$, both being well defined and positive for all $\mathrm{x}, \mathrm{y}, \mathrm{z} \in \mathbb{R}^{N}$ and all $r, s, t$ satisfying $r \in(s, t) \subset[0, T]$. For every $F \in \mathcal{B}_{N} \times \mathcal{B}_{N}$, let us also consider a positive measure $\mu \in \mathcal{M}\left(\mathbb{R}^{N} \times \mathbb{R}^{N}, \mathbb{C}\right)$ such that

$$
\mu(F):=\int_{F} d \mu(\mathrm{x}, \mathrm{y})
$$

defines a probability measure on $\mathcal{B}_{N} \times \mathcal{B}_{N}$, thus satisfying

$$
\int_{\mathbb{R}^{N} \times \mathbb{R}^{N}} d \mu(\mathrm{x}, \mathrm{y})=1
$$

The knowledge of both (13) and (14) then makes it possible to associate a Bernstein process with (1)-(2). The precise statement is the following:

Theorem 1. Assume that Hypothesis (H1) holds, and let $P$ and $\mu$ be given by (13) and (14)-(15), respectively. Then, there exists a probability space $\left(\Omega, \mathcal{F}, \mathbb{P}_{\mu}\right)$ supporting an $\mathbb{R}^{N}$-valued Bernstein process $Z_{\tau \in[0, T]}$ such that the following properties are valid: 
(a) The function $P$ is the transition function of $Z_{\tau \in[0, T]}$ in the sense that

$$
\mathbb{P}_{\mu}\left(Z_{r} \in E \mid Z_{s}, Z_{t}\right)=P\left(Z_{t}, t ; E, r ; Z_{s}, s\right)
$$

for each $E \in \mathcal{B}_{N}$ and all $r, s, t$ satisfying $r \in(s, t) \subset[0, T]$.

(b) For every $n \in \mathbb{N}^{+}$, the finite-dimensional distributions of the process are given by

$$
\begin{aligned}
& \mathbb{P}_{\mu}\left(Z_{t_{1}} \in E_{1}, \ldots, Z_{t_{n}} \in E_{n}\right) \\
= & \int_{\mathbb{R}^{N} \times \mathbb{R}^{N}} \frac{d \mu(\mathrm{x}, \mathrm{y})}{g(\mathrm{x}, T, \mathrm{y})} \int_{E_{1}} d \mathrm{x}_{1} \ldots \int_{E_{n}} d \mathrm{x}_{n} \\
& \times \prod_{k=1}^{n} g\left(\mathrm{x}_{k}, t_{k}-t_{k-1}, \mathrm{x}_{k-1}\right) \times g\left(\mathrm{y}, T-t_{n}, \mathrm{x}_{n}\right)
\end{aligned}
$$

for all $E_{1}, \ldots, E_{n} \in \mathcal{B}_{N}$ and all $t_{0}=0<t_{1}<\ldots<t_{n}<T$, where $\mathrm{x}_{0}=\mathrm{x}$. In particular we have

$$
\begin{aligned}
& \mathbb{P}_{\mu}\left(Z_{t} \in E\right) \\
= & \int_{\mathbb{R}^{N} \times \mathbb{R}^{N}} \frac{d \mu(\mathrm{x}, \mathrm{y})}{g(\mathrm{x}, T, \mathrm{y})} \int_{E} d \mathrm{z} g(\mathrm{x}, t, \mathrm{z}) g(\mathrm{z}, T-t, \mathrm{y})
\end{aligned}
$$

for each $E \in \mathcal{B}_{N}$ and every $t \in(0, T)$. Moreover,

$$
\mathbb{P}_{\mu}\left(Z_{0} \in E\right)=\mu\left(E \times \mathbb{R}^{N}\right)
$$

and

$$
\mathbb{P}_{\mu}\left(Z_{T} \in E\right)=\mu\left(\mathbb{R}^{N} \times E\right)
$$

for each $E \in \mathcal{B}_{N}$.

(c) $\mathbb{P}_{\mu}$ is the only probability measure leading to the above properties.

Proof. Up to minor technical details, a direct adaptation of the method developed in Section 2 of [21] leads to

$$
\begin{aligned}
& \mathbb{P}_{\mu}\left(Z_{0} \in E_{0}, Z_{t_{1}} \in E_{1}, \ldots, Z_{t_{n}} \in E_{n}, Z_{T} \in E_{T}\right) \\
= & \int_{E_{0} \times E_{T}} d \mu(\mathrm{x}, \mathrm{y}) \int_{E_{1}} d \mathrm{x}_{1} \ldots \int_{E_{n}} d \mathrm{x}_{n} \prod_{k=1}^{n} p\left(\mathrm{y}, T ; \mathrm{x}_{k}, t_{k} ; \mathrm{x}_{k-1}, t_{k-1}\right)
\end{aligned}
$$

for all $E_{0}, \ldots, E_{T} \in B_{N}$ and all $t_{0}=0<t_{1}<\ldots<t_{n}<T$, where $\mathrm{x}_{0}=\mathrm{x}$. In particular we have

$$
\mathbb{P}_{\mu}\left(Z_{0} \in E_{0}, Z_{T} \in E_{T}\right)=\mu\left(E_{0} \times E_{T}\right)
$$

for all $E_{0}, E_{T} \in B_{N}$, that is, (14) is the joint probability distribution of $Z_{0}$ and 
$Z_{T}$. Now, from (12) we obtain

$$
\begin{aligned}
& \prod_{k=1}^{n} p\left(\mathrm{y}, T ; \mathrm{x}_{k}, t_{k} ; \mathrm{x}_{k-1}, t_{k-1}\right) \\
= & \prod_{k=1}^{n} \frac{g\left(\mathrm{y}, T-t_{k}, \mathrm{x}_{k}\right) g\left(\mathrm{x}_{k}, t_{k}-t_{k-1}, \mathrm{x}_{k-1}\right)}{g\left(\mathrm{y}, T-t_{k-1}, \mathrm{x}_{k-1}\right)} \\
= & \frac{1}{g(\mathrm{x}, T, \mathrm{y})} \prod_{k=1}^{n} g\left(\mathrm{x}_{k}, t_{k}-t_{k-1}, \mathrm{x}_{k-1}\right) \times g\left(\mathrm{y}, T-t_{n}, \mathrm{x}_{n}\right)
\end{aligned}
$$

after $n-1$ cancellations of factors in order to obtain the second equality, so that (16) follows by choosing $E_{0}=E_{T}=\mathbb{R}^{N}$ in (20). Relations (18) and (19) are just a particular case of (21).

Of course, we can say more about $Z_{\tau \in[0, T]}$ if we know more about $\mu$. First, $Z_{\tau \in[0, T]}$ is Markovian if there exist positive measures $\nu_{0}, \nu_{T} \in \mathcal{M}\left(\mathbb{R}^{N}, \mathbb{C}\right)$ such that

$$
\mu(F)=\int_{F} d\left(\nu_{0} \otimes \nu_{T}\right)(\mathrm{x}, \mathrm{y}) g(\mathrm{x}, T, \mathrm{y})
$$

for every $F \in \mathcal{B}_{N} \times \mathcal{B}_{N}$, with

$$
\int_{\mathbb{R}^{N} \times \mathbb{R}^{N}} d\left(\nu_{0} \otimes \nu_{T}\right)(\mathrm{x}, \mathrm{y}) g(\mathrm{x}, T, \mathrm{y})=1
$$

in which case we also say that $\mu$ is Markovian. This result can be traced back to the more general Theorem 3.1 in [10], and allows us to make a closer connection between $Z_{\tau \in[0, T]}$ and (1)-(2) provided we impose the following hypothesis:

(H2) The measures $\varphi_{0}, \psi_{T} \in \mathcal{M}\left(\mathbb{R}^{N}, \mathbb{C}\right)$ in (1)-(2) are positive, and there exist a unique classical positive solution to (1) and a unique classical positive solution to (2), namely,

$$
u_{\varphi_{0}}(\mathrm{x}, t)=\int_{\mathbb{R}^{N}} d \varphi_{0}(\mathrm{y}) g(\mathrm{x}, t, \mathrm{y})
$$

and

$$
v_{\psi_{T}}(\mathrm{x}, t)=\int_{\mathbb{R}^{N}} d \psi_{T}(\mathrm{y}) g(\mathrm{x}, T-t, \mathrm{y})
$$

respectively.

We then have the following consequence of Theorem 1 :

Corollary 1. Assume that Hypotheses (H1)-(H2) hold, and let us choose $\mu$ of the form (22) with $\nu_{0}=\varphi_{0}$ and $\nu_{T}=\psi_{T}$. Then, the Bernstein process 
$Z_{\tau \in[0, T]}$ of Theorem 1 is Markovian, and its finite-dimensional distributions are given by

$$
\begin{aligned}
& \mathbb{P}_{\mu}\left(Z_{t_{1}} \in E_{1}, \ldots, Z_{t_{n}} \in E_{n}\right) \\
= & \int_{E_{1}} d \mathrm{x}_{1} \ldots \int_{E_{n}} d \mathrm{x}_{n} \prod_{k=2}^{n} g\left(\mathrm{x}_{k}, t_{k}-t_{k-1}, \mathrm{x}_{k-1}\right) \times u_{\varphi_{0}}\left(\mathrm{x}_{1}, t_{1}\right) v_{\psi_{T}}\left(\mathrm{x}_{n}, t_{n}\right)
\end{aligned}
$$

for every integer $n \geq 2$, all $E_{1}, \ldots, E_{n} \in \mathcal{B}_{N}$ and all $t_{0}=0<t_{1}<\ldots<t_{n}<T$, where $\mathrm{x}_{0}=\mathrm{x}$. Moreover we have

$$
\begin{aligned}
& \mathbb{P}_{\mu}\left(Z_{t} \in E\right) \\
= & \int_{E} d \times u_{\varphi_{0}}(\mathrm{x}, t) v_{\psi_{T}}(\mathrm{x}, t)
\end{aligned}
$$

for each $E \in \mathcal{B}_{N}$ and every $t \in(0, T)$. Finally,

$$
\mathbb{P}_{\mu}\left(Z_{0} \in E\right)=\int_{E} d \varphi_{0}(\mathrm{x}) v_{\psi_{T}}(\mathrm{x}, 0)
$$

and

$$
\mathbb{P}_{\mu}\left(Z_{T} \in E\right)=\int_{E} d \psi_{T}(\mathrm{x}) u_{\varphi_{0}}(\mathrm{x}, T)
$$

for each $E \in \mathcal{B}_{N}$.

Proof. We first rewrite (16) as

$$
\begin{aligned}
& \mathbb{P}_{\mu}\left(Z_{t_{1}} \in E_{1}, \ldots, Z_{t_{n}} \in E_{n}\right) \\
= & \int_{\mathbb{R}^{N} \times \mathbb{R}^{N}} \frac{d \mu(\mathrm{x}, \mathrm{y})}{g(\mathrm{x}, T, \mathrm{y})} \int_{E_{1}} d \mathrm{x}_{1} \ldots \int_{E_{n}} d \mathrm{x}_{n} \\
& \times \prod_{k=2}^{n} g\left(\mathrm{x}_{k}, t_{k}-t_{k-1}, \mathrm{x}_{k-1}\right) \times g\left(\mathrm{x}_{1}, t_{1}, \mathrm{x}\right) g\left(\mathrm{y}, T-t_{n}, \mathrm{x}_{n}\right)
\end{aligned}
$$

and then substitute the choice of the measure $\mu$ into (19) and (30), using (24)(25) along with the symmetry property of $g$ with respect to the spatial variables.

Remarks. (1) In the Markovian case, we have thus exhibited a very general class of initial-final conditions in (1)-(2) which allows us to determine the processes $Z_{\tau \in[0, T]}$ completely, including their marginal distributions (28)-(29). The converse point of view was developed in [2] and its references, where it was shown instead that it is a general class of marginal distributions which determines the initial-final data of the relevant partial differential equations, through a system of nonlinear integral equations. However, the resulting initial-final conditions of [2] belong to the class of positive continuous functions, and not to the larger class of positive measures as is the case in this article. 
(2) A glance at (25) shows that it is sufficient to use elementary time reversal in Green's function to obtain the solution to (2). Although the situation is not always that simple, particularly when the given parabolic equations are nonautonomous, it is still possible to define a quite appropriate probabilistic notion of time symmetry in general. We refer the reader to [21] for further details.

Relations (16) and (26) are the fundamental relations that will allow us to construct the Gaussian processes associated with (3)-(4) in the next sections.

\section{Two Ornstein-Uhlenbeck processes and a Bern- stein bridge}

In the remaining part of this article we denote by $(., .)_{\mathbb{R}^{N}}$ the Euclidean inner product in $\mathbb{R}^{N}$, and by $L^{2}\left(\mathbb{R}^{N}, \mathbb{C}\right)$ the usual Lebesgue space of all complexvalued, square-integrable functions on $\mathbb{R}^{N}$. We begin by considering the forwardbackward system (3)-(4) with centered Gaussian initial-final data, namely,

$$
\begin{aligned}
\partial_{t} u(\mathrm{x}, t) & =\frac{1}{2} \Delta_{\mathrm{x}} u(\mathrm{x}, t)-\frac{\lambda^{2}|\mathrm{x}|^{2}}{2} u(\mathrm{x}, t), \quad(\mathrm{x}, t) \in \mathbb{R}^{N} \times(0, T] \\
u(\mathrm{x}, 0) & =\varphi_{0, \lambda}(\mathrm{x})=\left(\frac{\lambda \exp [\lambda T]}{\pi}\right)^{\frac{N}{4}} \exp \left[-\frac{\lambda|\mathrm{x}|^{2}}{2}\right]
\end{aligned}
$$

and

$$
\begin{aligned}
-\partial_{t} v(\mathrm{x}, t) & =\frac{1}{2} \Delta_{\mathrm{x}} v(\mathrm{x}, t)-\frac{\lambda^{2}|\mathrm{x}|^{2}}{2} v(\mathrm{x}, t), \quad(\mathrm{x}, t) \in \mathbb{R}^{N} \times[0, T), \\
v(\mathrm{x}, T) & =\psi_{T, \lambda}(\mathrm{x})=\left(\frac{\lambda \exp [\lambda T]}{\pi}\right)^{\frac{N}{4}} \exp \left[-\frac{\lambda|\mathrm{x}|^{2}}{2}\right],
\end{aligned}
$$

thereby identifying the measure $\varphi_{0, \lambda}=\psi_{T, \lambda}$ with its Gaussian density relative to the Lebesgue measure in $\mathbb{R}^{N}$. Let us recall that the self-adjoint realization in $L^{2}\left(\mathbb{R}^{N}, \mathbb{C}\right)$ of the elliptic operator on the right-hand side of these equations has a pure point spectrum. More specifically, for every $m \in \mathbb{N}$ let

$$
h_{m, \lambda}(x):=\sqrt[4]{\lambda} h_{m}(\sqrt{\lambda} x)
$$

be the one-dimensional, suitably scaled Hermite functions where

$$
h_{m}(x)=\left(\pi^{\frac{1}{2}} 2^{m} m !\right)^{-\frac{1}{2}} e^{-\frac{x^{2}}{2}} H_{m}(x),
$$

and where the $H_{m}$ 's are the Hermite polynomials

$$
H_{m}(x)=(-1)^{m} e^{x^{2}} \frac{d^{m}}{d x^{m}} e^{-x^{2}} .
$$


The following spectral result regarding the operator on the right-hand side of (31) is well-known and in any case can be verified directly by means of an explicit computation:

Lemma 1. The tensor products $\otimes_{j=1}^{N} h_{m_{j}, \lambda}$ where the $m_{j}$ 's vary independently on $\mathbb{N}$ provide an orthonormal basis of $L^{2}\left(\mathbb{R}^{N}, \mathbb{C}\right)$, and moreover the eigenvalue equation

$$
\left(-\frac{1}{2} \Delta_{\mathrm{x}}+\frac{\lambda^{2}|\mathrm{x}|^{2}}{2}\right) \otimes_{j=1}^{N} h_{m_{j}, \lambda}(\mathrm{x})=\lambda\left(\sum_{j=1}^{N} m_{j}+\frac{N}{2}\right) \otimes_{j=1}^{N} h_{m_{j}, \lambda}(\mathrm{x})
$$

holds for every $\mathrm{x} \in \mathbb{R}^{N}$.

In particular, by reference to (34) and (35) we have

$$
\otimes_{j=1}^{N} h_{0, \lambda}(\mathrm{x})=\left(\frac{\lambda}{\pi}\right)^{\frac{N}{4}} \exp \left[-\frac{\lambda|\mathrm{x}|^{2}}{2}\right]>0
$$

whose associated eigenvalue is $E_{\lambda}=\frac{N \lambda}{2}$, so that (37) corresponds to the initialfinal conditions in (31)-(32) up to a normalization factor chosen in such a way that (15) holds for the measure

$$
\mu_{\lambda}(F)=\int_{F} d x d y \varphi_{0, \lambda}(\mathrm{x}) \psi_{T, \lambda}(\mathrm{y}) g_{\lambda}(\mathrm{x}, T, \mathrm{y})
$$

where $F \in \mathcal{B}_{N} \times \mathcal{B}_{N}$, and where

$$
\begin{aligned}
& g_{\lambda}(\mathrm{x}, t, \mathrm{y}) \\
= & \left(\frac{\lambda}{2 \pi \sinh (\lambda t)}\right)^{\frac{N}{2}} \exp \left[-\frac{\lambda\left(\cosh (\lambda t)\left(|\mathrm{x}|^{2}+|\mathrm{y}|^{2}\right)-2(\mathrm{x}, \mathrm{y})_{\mathbb{R}^{N}}\right)}{2 \sinh (\lambda t)}\right]
\end{aligned}
$$

is the $N$-dimensional version of Mehler's kernel for $t \in(0, T]$ (see the appendix for details). In this way, the unique classical positive solutions to (31)-(32) satisfying the requirement

$$
\int_{\mathbb{R}^{N}} d \times u_{\lambda}(\mathrm{x}, t) v_{\lambda}(\mathrm{x}, t)=1
$$

for every $t \in[0, T]$ are

$$
u_{\lambda}(\mathrm{x}, t)=\left(\frac{\lambda \exp [\lambda T]}{\pi}\right)^{\frac{N}{4}} \exp \left[-\frac{\lambda\left(|\mathrm{x}|^{2}+N t\right)}{2}\right]
$$

and

$$
v_{\lambda}(\mathrm{x}, t)=\left(\frac{\lambda \exp [\lambda T]}{\pi}\right)^{\frac{N}{4}} \exp \left[-\frac{\lambda\left(|\mathrm{x}|^{2}+N(T-t)\right)}{2}\right]
$$


respectively. Then the following result holds, where $\mathbb{E}_{\mu}$ stands for the expectation functional on $\left(\Omega, \mathcal{F}, \mathbb{P}_{\mu}\right)$ :

Proposition 1. The Bernstein process $Z_{\tau \in[0, T]}^{\lambda}$ associated with (31)-(32) in the sense of Corollary 1 is a Gaussian and Markovian process such that

$$
\mathbb{P}_{\mu}\left(Z_{t}^{\lambda} \in E\right)=\left(2 \pi \sigma_{\lambda}\right)^{-\frac{N}{2}} \int_{E} d x \exp \left[-\frac{|\mathrm{x}|^{2}}{2 \sigma_{\lambda}}\right]
$$

for each $t \in[0, T]$ and every $E \in \mathcal{B}_{N}$, where

$$
\sigma_{\lambda}=\frac{1}{2 \lambda}
$$

Furthermore, the components of $Z_{\tau \in[0, T]}^{\lambda}$ satisfy the relation

$$
\mathbb{E}_{\mu}\left(Z_{s}^{\lambda, i} Z_{t}^{\lambda, j}\right)=\frac{\exp [-\lambda|t-s|]}{2 \lambda} \delta_{i, j}
$$

for all $s, t \in[0, T]$ and all $i, j \in\{1, \ldots, N\}$. Thus, $Z_{\tau \in[0, T]}^{\lambda}$ identifies in law with a process whose components are all independent, one-dimensional and stationary Ornstein-Uhlenbeck processes.

Proof. The fact that $Z_{\tau \in[0, T]}^{\lambda}$ is Gaussian and Markovian satisfying (42) follows from Corollary 1 with (38) and (40)-(41) plugged into (26) and (27), so that the density of the probability distribution for $\left(Z_{t_{1}}^{\lambda}, \ldots, Z_{t_{n}}^{\lambda}\right) \in \mathbb{R}^{n N}$ is

$$
\begin{aligned}
& \prod_{k=2}^{n} g_{\lambda}\left(\mathrm{x}_{k}, t_{k}-t_{k-1}, \mathrm{x}_{k-1}\right) \times u_{\lambda}\left(\mathrm{x}_{1}, t_{1}\right) v_{\lambda}\left(\mathrm{x}_{n}, t_{n}\right) \\
= & (2 \pi)^{-\frac{n N}{2}}\left(2 \lambda^{n} e^{\lambda\left(t_{n}-t_{1}\right)}\right)^{\frac{N}{2}}\left(\prod_{k=2}^{n} \sinh \left(\lambda\left(t_{k}-t_{k-1}\right)\right)\right)^{-\frac{N}{2}} \\
& \times \exp \left[-\frac{\lambda}{2} \sum_{k=2}^{n} \frac{\cosh \left(\lambda\left(t_{k}-t_{k-1}\right)\right)\left(\left|\mathrm{x}_{k}\right|^{2}+\left|\mathrm{x}_{k-1}\right|^{2}\right)-2\left(\mathrm{x}_{k}, \mathrm{x}_{k-1}\right)_{\mathbb{R}^{N}}}{\sinh \left(\lambda\left(t_{k}-t_{k-1}\right)\right)}\right] \\
& \times \exp \left[-\frac{\lambda}{2}\left(\left|\mathrm{x}_{1}\right|^{2}+\left|\mathrm{x}_{n}\right|^{2}\right)\right] .
\end{aligned}
$$

The $n N \times n N$ corresponding covariance matrix is then of the form $C_{\lambda} \otimes \mathbb{I}_{N}$ with $\mathbb{I}_{N}$ the identity matrix in $\mathbb{R}^{N}$, where $C_{\lambda}^{-1}$ is tridiagonal and obtained by identification of the quadratic form in the argument of the above exponentials when $N=1$. This gives

$$
C_{\lambda, k, k}^{-1}=\left\{\begin{array}{cc}
\frac{\lambda e^{\lambda\left(t_{2}-t_{1}\right)}}{\sinh \left(\lambda\left(t_{2}-t_{1}\right)\right)} & \text { for } k=1, \\
\frac{\lambda \sinh \left(\lambda\left(t_{k+1}-t_{k-1}\right)\right)}{\sinh \left(\lambda\left(t_{k+1}-t_{k}\right)\right) \sinh \left(\lambda\left(t_{k}-t_{k-1}\right)\right)} & \text { for } k=2, \ldots, n-1, \\
\frac{\lambda e^{\lambda\left(t_{n}-t_{n-1}\right)}}{\sinh \left(\lambda\left(t_{n}-t_{n-1}\right)\right)} & \text { for } k=n
\end{array}\right.
$$


(the second line not being there when $n=2$ ), and

$$
C_{\lambda, k, k-1}^{-1}=C_{\lambda, k-1, k}^{-1}=-\frac{\lambda}{\sinh \left(\lambda\left|t_{k}-t_{k-1}\right|\right)} \quad \text { for } k=2, \ldots, n .
$$

Consequently, using for instance the analytical inversion formulae in [9], or by direct verification, we obtain

$$
C_{\lambda, k, l}=\frac{\exp \left[-\lambda\left|t_{k}-t_{l}\right|\right]}{2 \lambda}
$$

for all $k, l \in\{1, \ldots, n\}$, so that (43) eventually holds. Now, let us consider the forward Itô integral equation

$$
\begin{aligned}
& X_{t}=e^{-\lambda t} Z_{0}^{\lambda}+\int_{0}^{t} e^{-\lambda(t-\tau)} d W_{\tau}, \quad t \in[0, T], \\
& X_{0}=Z_{0}^{\lambda}
\end{aligned}
$$

where $W_{\tau \in[0, T]}$ is a given Wiener process in $\mathbb{R}^{N}$, and where $Z_{0}^{\lambda}$ is distributed according to (42) and independent of $W_{\tau \in[0, T]}$. It is well known that the solution process $X_{\tau \in[0, T]}^{\lambda}$ to (44) is centered Gaussian with covariance (43) (see, e.g., Section 5.6 in Chapter 5 of [11]), so that $Z_{\tau \in[0, T]}^{\lambda}$ identifies in law with $X_{\tau \in[0, T]}^{\lambda}$. Therefore, $Z_{\tau \in[0, T]}^{\lambda}$ is indeed a $N$-dimensional Ornstein-Uhlenbeck process with the stated properties.

Next, we show that if we require instead $Z_{0}$ to be a given point in $\mathbb{R}^{N}$, the solution process to (44) is also a particular Bernstein process. We do this in the simplest case where $Z_{0}$ is the origin, by considering the forward-backward system

$$
\begin{aligned}
\partial_{t} u(\mathrm{x}, t) & =\frac{1}{2} \Delta_{\mathrm{x}} u(\mathrm{x}, t)-\frac{\lambda^{2}|\mathrm{x}|^{2}}{2} u(\mathrm{x}, t), \quad(\mathrm{x}, t) \in \mathbb{R}^{N} \times(0, T], \\
u(\mathrm{x}, 0) & =\varphi_{0, \lambda}(\mathrm{x})=(\exp [\lambda T])^{\frac{N}{4}} \delta(\mathrm{x})
\end{aligned}
$$

and

$$
\begin{aligned}
-\partial_{t} v(\mathrm{x}, t) & =\frac{1}{2} \Delta_{\mathrm{x}} v(\mathrm{x}, t)-\frac{\lambda^{2}|\mathrm{x}|^{2}}{2} v(\mathrm{x}, t), \quad(\mathrm{x}, t) \in \mathbb{R}^{N} \times[0, T), \\
v(\mathrm{x}, T) & =\psi_{T, \lambda}(\mathrm{x})=(\exp [\lambda T])^{\frac{N}{4}} \exp \left[-\frac{\lambda|\mathrm{x}|^{2}}{2}\right],
\end{aligned}
$$

with $\delta$ the Dirac measure. The corresponding Markovian measure (14) is then determined by

$$
\mu_{\lambda}(\mathrm{x}, \mathrm{y})=(\exp [\lambda T])^{\frac{N}{2}} \delta(\mathrm{x}) \exp \left[-\frac{\lambda|\mathrm{y}|^{2}}{2}\right] g(\mathrm{x}, T, \mathrm{y})
$$


and satisfies (15), while the unique relevant classical positive solutions to (45)(46) are

$$
u_{\lambda}(\mathrm{x}, t)=\left(\frac{\lambda \exp \left[\frac{\lambda T}{2}\right]}{2 \pi \sinh (\lambda t)}\right)^{\frac{N}{2}} \exp \left[-\frac{\lambda \operatorname{coth}(\lambda t)|\mathrm{x}|^{2}}{2}\right]
$$

and

$$
v_{\lambda}(\mathrm{x}, t)=(\exp [\lambda T])^{\frac{N}{4}} \exp \left[-\frac{\lambda\left(|\mathrm{x}|^{2}+N(T-t)\right)}{2}\right],
$$

respectively. We then have the following result:

Proposition 2. The Bernstein process $Z_{\tau \in[0, T]}^{\lambda}$ associated with (45)-(46) in the sense of Corollary 1 is a Gaussian and Markovian process such that

$$
\mathbb{P}_{\mu}\left(Z_{t}^{\lambda} \in E\right)=\left(2 \pi \sigma_{\lambda}(t)\right)^{-\frac{N}{2}} \int_{E} d x \exp \left[-\frac{|x|^{2}}{2 \sigma_{\lambda}(t)}\right]
$$

for each $t \in(0, T]$ and every $E \in \mathcal{B}_{N}$, where

$$
\sigma_{\lambda}(t)=\frac{\sinh (\lambda t) \exp [-\lambda t]}{\lambda} .
$$

Furthermore we have

$$
\mathbb{P}_{\mu}\left(Z_{0}^{\lambda}=\mathrm{o}\right)=1,
$$

and the components of $Z_{\tau \in[0, T]}^{\lambda}$ satisfy the relation

$$
\mathbb{E}_{\mu}\left(Z_{s}^{\lambda, i} Z_{t}^{\lambda, j}\right)=\frac{\exp [-\lambda(t+s)]}{2 \lambda}(\exp [2 \lambda(t \wedge s)]-1) \delta_{i, j}
$$

for all $s, t \in[0, T]$ and all $i, j \in\{1, \ldots, N\}$. Thus, $Z_{\tau \in[0, T]}^{\lambda}$ identifies in law with a process whose components are all independent, one-dimensional non-stationary Ornstein-Uhlenbeck processes conditioned to start at the origin.

Proof. As in the proof of Proposition 1, the first part of the statement including (49)-(51) follows from the appropriate substitutions into the formulae of Corollary 1. Furthermore, the matrix $C_{\lambda}^{-1}$ resulting from the identification of the quadratic form in the Gaussian density of $\left(Z_{t_{1}}^{\lambda}, \ldots, Z_{t_{n}}^{\lambda}\right)$ is the same as that in Proposition 1, with the exception of $C_{\lambda 1,1}^{-1}$ which now reads

$$
C_{\lambda, 1,1}^{-1}=\frac{\lambda \sinh \left(\lambda t_{2}\right)}{\sinh \left(\lambda\left(t_{2}-t_{1}\right)\right) \sinh \left(\lambda t_{1}\right)} .
$$

Inverting again we eventually obtain

$$
C_{\lambda, k, l}=\frac{\exp \left[-\lambda\left(t_{k}+t_{l}\right)\right]}{2 \lambda}\left(\exp \left[2 \lambda\left(t_{k} \wedge t_{l}\right)\right]-1\right)
$$


for all $k, l \in\{1, \ldots, n\}$, so that (52) holds. Therefore, $Z_{\tau \in[0, T]}^{\lambda}$ is indeed a $N$ dimensional Ornstein-Uhlenbeck process with the stated properties.

REMARKS. (1) It is equally easy to condition the Ornstein-Uhlenbeck process so that it ends at the origin of $\mathbb{R}^{N}$ when $t=T$. The underlying Bernstein process $\hat{Z}_{\tau \in[0, T]}^{\lambda}$ is then simply determined by swaping the initial-final conditions in (45) and (46). Indeed, in doing so the relevant solutions become

$$
u_{\lambda}(\mathrm{x}, t)=(\exp [\lambda T])^{\frac{N}{4}} \exp \left[-\frac{\lambda\left(|\mathrm{x}|^{2}+N t\right)}{2}\right]
$$

and

$$
v_{\lambda}(\mathrm{x}, t)=\left(\frac{\lambda \exp \left[\frac{\lambda T}{2}\right]}{2 \pi \sinh (\lambda(T-t))}\right)^{\frac{N}{2}} \exp \left[-\frac{\lambda \operatorname{coth}(\lambda(T-t))|\mathrm{x}|^{2}}{2}\right]
$$

instead of (47) and (48), respectively, so that $\hat{Z}_{\tau \in[0, T]}^{\lambda}$ is just the time-reversal of the process of Proposition 2, namely,

$$
\hat{Z}_{\tau}^{\lambda}=Z_{T-\tau}^{\lambda}
$$

for every $\tau \in[0, T]$.

(2) Whereas the Bernstein process of Proposition 1 is stationary, that of Proposition 2 is not. This is intuitively understandable, as some kind of non trivial dynamics ought to be necessary to steer the process from a deterministic state at $t=0$ to a Gaussian distribution at the end of its journey.

Of course, there is an almost unlimited number of possibilities of getting various Bernstein processes in the above manner, just by choosing $\varphi_{0, \lambda}$ and $\psi_{T, \lambda}$ appropriately. We complete this section by constructing yet another process which shares many properties of a Markovian bridge. For this, we consider the forward-backward system (3)-(4) with Dirac measures concentrated at the origin and at a given point $a \in \mathbb{R}^{N}$ as initial-final data, namely,

$$
\begin{aligned}
\partial_{t} u(\mathrm{x}, t) & =\frac{1}{2} \Delta_{\mathrm{x}} u(\mathrm{x}, t)-\frac{\lambda^{2}|\mathrm{x}|^{2}}{2} u(\mathrm{x}, t), \quad(\mathrm{x}, t) \in \mathbb{R}^{N} \times(0, T], \\
u(\mathrm{x}, 0) & =\varphi_{0, \lambda}(\mathrm{x})=\mathrm{m}_{\lambda} \delta(\mathrm{x})
\end{aligned}
$$

and

$$
\begin{aligned}
-\partial_{t} v(\mathrm{x}, t) & =\frac{1}{2} \Delta_{\mathrm{x}} v(\mathrm{x}, t)-\frac{\lambda^{2}|\mathrm{x}|^{2}}{2} v(\mathrm{x}, t), \quad(\mathrm{x}, t) \in \mathbb{R}^{N} \times[0, T), \\
v(\mathrm{x}, T) & =\psi_{T, \lambda}(\mathrm{x})=\mathrm{m}_{\lambda} \delta(\mathrm{x}-\mathrm{a}),
\end{aligned}
$$

where

$$
\mathrm{m}_{\lambda}=\left(\frac{2 \pi \sinh (\lambda T)}{\lambda}\right)^{\frac{N}{4}} \exp \left[\frac{\lambda \operatorname{coth}(\lambda T)|\mathrm{a}|^{2}}{4}\right]
$$


It is easily verified that the normalization condition

$$
\mathrm{m}_{\lambda}^{2} \int_{\mathbb{R}^{N} \times \mathbb{R}^{N}} d \times d y \delta(\mathrm{x}) \delta(\mathrm{y}-\mathrm{a}) g_{\lambda}(\mathrm{x}, T, \mathrm{y})=1
$$

holds with Mehler's kernel given above, and that the unique classical positive solutions to (53)-(54) which satisfy (39) are now

$$
u_{\lambda}(\mathrm{x}, t)=\mathrm{n}_{\lambda} \sinh ^{-\frac{N}{2}}(\lambda t) \exp \left[-\frac{\alpha_{\lambda}(t)|\mathrm{x}|^{2}}{2}\right]
$$

and

$$
\begin{aligned}
v_{\lambda}(\mathrm{x}, t)= & \mathrm{n}_{\lambda} \exp \left[-\frac{\alpha_{\lambda}(T-t)|\mathrm{a}|^{2}}{2}\right] \sinh ^{-\frac{N}{2}}(\lambda(T-t)) \\
& \times \exp \left[-\frac{1}{2}\left(\alpha_{\lambda}(T-t)|\mathrm{x}|^{2}-\frac{2 \lambda(\mathrm{a}, \mathrm{x})_{\mathbb{R}^{N}}}{\sinh (\lambda(T-t))}\right)\right],
\end{aligned}
$$

respectively, where

$$
\alpha_{\lambda}(t)=\lambda \operatorname{coth}(\lambda t)
$$

and

$$
\mathrm{n}_{\lambda}=\left(\frac{\lambda \sinh (\lambda T)}{2 \pi}\right)^{\frac{N}{4}} \exp \left[\frac{\alpha_{\lambda}(T)|\mathrm{a}|^{2}}{4}\right] .
$$

Then the following result is valid:

Proposition 3. The Bernstein process $Z_{\tau \in[0, T]}^{\lambda}$ associated with (53)-(54) in the sense of Corollary 1 is a Gaussian and Markovian process such that

$$
\mathbb{P}_{\mu}\left(Z_{t}^{\lambda} \in E\right)=\left(2 \pi \sigma_{\lambda}(t)\right)^{-\frac{N}{2}} \int_{E} d x \exp \left[-\frac{\left|\mathrm{x}-\mathrm{a}_{\lambda}(t)\right|^{2}}{2 \sigma_{\lambda}(t)}\right]
$$

for each $t \in(0, T)$ and every $E \in \mathcal{B}_{N}$, where

$$
\mathrm{a}_{\lambda}(t)=\frac{\sinh (\lambda t)}{\sinh (\lambda T)} \mathrm{a}
$$

and

$$
\sigma_{\lambda}(t)=\frac{\sinh (\lambda(T-t)) \sinh (\lambda t)}{\lambda \sinh (\lambda T)}
$$

Furthermore we have

$$
\begin{aligned}
& \mathbb{P}_{\mu}\left(Z_{0}^{\lambda}=\mathrm{o}\right) \\
= & \mathbb{P}_{\mu}\left(Z_{T}^{\lambda}=\mathrm{a}\right)=1,
\end{aligned}
$$


and the components of $Z_{\tau \in[0, T]}^{\lambda}$ satisfy the relation

$$
\mathbb{E}_{\mu}\left(\left(Z_{s}^{\lambda, i}-a_{\lambda}^{i}(s)\right)\left(Z_{t}^{\lambda, j}-a_{\lambda}^{j}(t)\right)\right)= \begin{cases}\frac{\sinh (\lambda(T-t)) \sinh (\lambda s)}{\lambda \sinh (\lambda T)} \delta_{i, j} & \text { for } t \geq s \\ \frac{\sinh (\lambda(T-s)) \sinh (\lambda t)}{\lambda \sinh (\lambda T)} \delta_{i, j} & \text { for } t \leq s\end{cases}
$$

for all $s, t \in[0, T]$ and all $i, j \in\{1, \ldots, N\}$. In fact, $Z_{\tau \in[0, T]}^{\lambda}$ is a non-stationary process pinned down at the origin when $t=0$, at a when $t=T$, and exhibiting maximal randomness when $t=\frac{T}{2}$.

Proof. We begin by proving (60). Using (55), (56) and (59) we first have

$$
\begin{aligned}
& u_{\lambda}(\mathrm{x}, t) v_{\lambda}(\mathrm{x}, t) \\
= & \left(\frac{\lambda \sinh (\lambda T)}{2 \pi \sinh (\lambda(T-t)) \sinh (\lambda t)}\right)^{\frac{N}{2}} \exp \left[\frac{\left(\alpha_{\lambda}(T)-\alpha_{\lambda}(T-t)\right)|\mathrm{a}|^{2}}{2}\right] \\
& \times \exp \left[-\frac{1}{2}\left(\left(\alpha_{\lambda}(t)+\alpha_{\lambda}(T-t)\right)|\mathrm{x}|^{2}-\frac{2 \lambda(\mathrm{a}, \mathrm{x})_{\mathbb{R}^{N}}}{\sinh (\lambda(T-t))}\right)\right]
\end{aligned}
$$

after regrouping terms, and furthermore

$$
\begin{aligned}
\alpha_{\lambda}(T)-\alpha_{\lambda}(T-t) & =-\frac{\lambda \sinh (\lambda t)}{\sinh (\lambda(T-t)) \sinh (\lambda T)} \\
\alpha_{\lambda}(t)+\alpha_{\lambda}(T-t) & =\frac{\lambda \sinh (\lambda T)}{\sinh (\lambda(T-t)) \sinh (\lambda t)}
\end{aligned}
$$

from (58). The substitution of (66)-(67) into (65) then leads to

$$
\begin{aligned}
& u_{\lambda}(\mathrm{x}, t) v_{\lambda}(\mathrm{x}, t) \\
= & \left(\frac{\lambda \sinh (\lambda T)}{2 \pi \sinh (\lambda(T-t)) \sinh (\lambda t)}\right)^{\frac{N}{2}} \exp \left[-\frac{\lambda \sinh (\lambda t)|\mathrm{a}|^{2}}{2 \sinh (\lambda(T-t)) \sinh (\lambda T)}\right] \\
& \times \exp \left[-\frac{\lambda}{2}\left(\frac{\sinh (\lambda T)|\mathrm{x}|^{2}-2 \sinh (\lambda t)(\mathrm{a}, \mathrm{x})_{\mathbb{R}^{N}}}{\sinh (\lambda(T-t)) \sinh (\lambda t)}\right)\right] .
\end{aligned}
$$

Now, for the numerator of the argument in the second exponential of (68) we have

$$
\begin{gathered}
\sinh (\lambda T)|x|^{2}-2 \sinh (\lambda t)(\mathrm{a}, \mathrm{x})_{\mathbb{R}^{N}} \\
=\sinh (\lambda T)\left|\mathrm{x}-\mathrm{a}_{\lambda}(t)\right|^{2}-\frac{\sinh ^{2}(\lambda t)|\mathrm{a}|^{2}}{\sinh (\lambda T)}
\end{gathered}
$$

by virtue of (61). Therefore, taking (62) and (69) into account in (68) we get

$$
\begin{aligned}
& u_{\lambda}(\mathrm{x}, t) v_{\lambda}(\mathrm{x}, t) \\
= & \left(2 \pi \sigma_{\lambda}(t)\right)^{-\frac{N}{2}} \exp \left[-\frac{\left|\mathrm{x}-\mathrm{a}_{\lambda}(t)\right|^{2}}{2 \sigma_{\lambda}(t)}\right]
\end{aligned}
$$


following the cancellation of two exponential factors, which gives the desired result according to (27).

As for the proof of (63), we remark that (28) and (29) lead to

$$
\begin{aligned}
& \mathbb{P}_{\mu}\left(Z_{0} \in E\right) \\
= & \int_{E} d \times \delta(\mathrm{x}) \exp \left[-\frac{\lambda\left(\cosh (\lambda T)|\mathrm{x}|^{2}-2(\mathrm{a}, \mathrm{x})_{\mathbb{R}^{N}}\right)}{2 \sinh (\lambda T)}\right]
\end{aligned}
$$

and

$$
\begin{aligned}
& \mathbb{P}_{\mu}\left(Z_{T} \in E\right) \\
= & \exp \left[\frac{\alpha_{\lambda}(T)|\mathrm{a}|^{2}}{2}\right] \int_{E} d \times \delta(\mathrm{x}-\mathrm{a}) \exp \left[-\frac{\alpha_{\lambda}(T)|\mathrm{x}|^{2}}{2}\right]
\end{aligned}
$$

for every $E \in \mathcal{B}_{N}$, respectively, which immediately imply the claim.

We now turn to the proof of (64), by noticing that in this case the finitedimensional density in $\mathbb{R}^{n N}$ is

$$
\begin{aligned}
& \prod_{k=2}^{n} g_{\lambda}\left(\mathrm{x}_{k}, t_{k}-t_{k-1}, \mathrm{x}_{k-1}\right) \times u_{\lambda}\left(\mathrm{x}_{1}, t_{1}\right) v_{\lambda}\left(\mathrm{x}_{n}, t_{n}\right) \\
= & (2 \pi)^{-\frac{n N}{2}}\left(\frac{\lambda^{n} \sinh (\lambda T)}{\sinh \left(\lambda\left(T-t_{n}\right)\right) \sinh \left(\lambda t_{1}\right)}\right)^{\frac{N}{2}}\left(\prod_{k=2}^{n} \sinh \left(\lambda\left(t_{k}-t_{k-1}\right)\right)\right)^{-\frac{N}{2}} \\
& \times \exp \left[\frac{1}{2}\left(\alpha_{\lambda}(T)-\alpha_{\lambda}\left(T-t_{n}\right)\right)|\mathrm{a}|^{2}\right] \\
& \times \exp \left[-\frac{\lambda}{2} \sum_{k=2}^{n} \frac{\cosh \left(\lambda\left(t_{k}-t_{k-1}\right)\right)\left(\left|\mathrm{x}_{k}\right|^{2}+\left|\mathrm{x}_{k-1}\right|^{2}\right)-2\left(\mathrm{x}_{k}, \mathrm{x}_{k-1}\right)_{\mathbb{R}^{N}}}{\sinh \left(\lambda\left(t_{k}-t_{k-1}\right)\right)}\right. \\
& \times \exp \left[-\frac{1}{2}\left(\alpha_{\lambda}\left(t_{1}\right)\left|\mathrm{x}_{1}\right|^{2}+\alpha_{\lambda}\left(T-t_{n}\right)\left|\mathrm{x}_{n}\right|^{2}\right)\right] \times \exp \left[\frac{\lambda\left(\mathrm{a}, \mathrm{x}_{n}\right)_{\mathbb{R}^{N}}}{\sinh \left(\lambda\left(T-t_{n}\right)\right)}\right] .
\end{aligned}
$$

Therefore, for the tridiagonal matrix $C_{\lambda}^{-1}$ corresponding to the quadratic part when $N=1$ we obtain

$$
C_{\lambda, k, k}^{-1}=\left\{\begin{array}{cl}
\frac{\lambda \sinh \left(\lambda t_{2}\right)}{\sinh \left(\lambda\left(t_{2}-t_{1}\right)\right) \sinh \left(\lambda t_{1}\right)} & \text { for } k=1, \\
\frac{\lambda \sinh \left(\lambda\left(t_{k+1}-t_{k-1}\right)\right)}{\sinh \left(\lambda\left(t_{k+1}-t_{k}\right)\right) \sinh \left(\lambda\left(t_{k}-t_{k-1}\right)\right)} & \text { for } k=2, \ldots, n-1, \\
\frac{\lambda \sinh \left(\lambda\left(T-t_{n-1}\right)\right)}{\sinh \left(\lambda\left(T-t_{n}\right)\right) \sinh \left(\lambda\left(t_{n}-t_{n-1}\right)\right)} & \text { for } k=n
\end{array}\right.
$$

(the second line still not being there if $n=2$ ), and

$$
C_{\lambda, k, k-1}^{-1}=C_{\lambda, k-1, k}^{-1}=-\frac{\lambda}{\sinh \left(\lambda\left|t_{k}-t_{k-1}\right|\right)} \quad \text { for } k=2, \ldots, n .
$$


Consequently, inverting again and using numerous relations among hyperbolic functions we eventually get

$$
C_{\lambda, k, l}= \begin{cases}\frac{\sinh \left(\lambda\left(T-t_{k}\right) \sinh \left(\lambda t_{l}\right)\right.}{\lambda \sinh (\lambda T)} & \text { for } k \geq l \\ \frac{\sinh \left(\lambda\left(T-t_{l}\right)\right) \sinh \left(\lambda t_{k}\right)}{\lambda \sinh (\lambda T)} & \text { for } k \leq l\end{cases}
$$

which leads to (64) by standard arguments. Finally, we note that the curve $\sigma_{\lambda}$ : $[0, T] \mapsto \mathbb{R}_{0}^{+}$given by $(62)$ is concave aside from satisfying $\sigma_{\lambda}(0)=\sigma_{\lambda}(T)=0$, and that it takes on the maximal value

$$
\sigma_{\lambda}\left(\frac{T}{2}\right)=\frac{\sinh ^{2}\left(\frac{\lambda T}{2}\right)}{\lambda \sinh (\lambda T)} .
$$

Thus, the process $Z_{\tau \in[0, T]}^{\lambda}$ is indeed non-stationary with the stated properties.

REMARK. We may dub the process $Z_{\tau \in[0, T]}^{\lambda}$ of the preceding corollary a Bernstein bridge, which represents a random curve whose ends are pinned down at specified points in space. We remark that the corresponding Gaussian law is no longer centered, unless $\mathrm{a}=\mathrm{o}$ in which case the process materializes a Markovian loop which retains the main features of a Brownian loop. In fact, $Z_{\tau \in[0, T]}^{\lambda}$ does reduce to a Brownian bridge in the limit $\lambda \rightarrow 0_{+}$since

$$
\lim _{\lambda \rightarrow 0_{+}} \mathbb{E}_{\mu}\left(\left(Z_{s}^{\lambda, i}-a_{\lambda}^{i}(s)\right)\left(Z_{t}^{\lambda, j}-a_{\lambda}^{j}(t)\right)\right)= \begin{cases}\frac{(T-t) s}{T} \delta_{i, j} & \text { for } t \geq s, \\ \frac{(T-s) t}{T} \delta_{i, j} & \text { for } t \leq s\end{cases}
$$

according to (64).

In the next section we introduce a new class of Bernstein processes which we can eventually relate to the so-called periodic Ornstein-Uhlenbeck process, and which is generated by a one-parameter family of non-Markovian probability measures.

\section{A family of non-Markovian Bernstein processes and the periodic Ornstein-Uhlenbeck process}

The Bernstein processes of this section are still defined from measures which are intimately tied up with problems of the form (3)-(4), but their finite-dimensional distributions will be determined exclusively from Theorem 1 . Instead of considering just one pair of parabolic problems such as (3)-(4), we first introduce an infinite hierarchy of forward-backward systems of the form

$$
\begin{aligned}
\partial_{t} u(\mathrm{x}, t) & =\frac{1}{2} \Delta_{\mathrm{x}} u(\mathrm{x}, t)-\frac{\lambda^{2}}{2}|\mathrm{x}|^{2} u(\mathrm{x}, t), \quad(\mathrm{x}, t) \in \mathbb{R}^{N} \times(0, T], \\
u(\mathrm{x}, 0) & =\varphi_{\mathrm{m}, 0, \lambda}(\mathrm{x})=e^{\frac{1}{2}\left(\sum_{j=1}^{N} m_{j}+\frac{N}{2}\right) \lambda T} \otimes_{j=1}^{N} h_{m_{j}, \lambda}(\mathrm{x}), \quad \mathrm{x} \in \mathbb{R}^{N}
\end{aligned}
$$


and

$$
\begin{aligned}
-\partial_{t} v(\mathrm{x}, t) & =\frac{1}{2} \Delta_{\mathrm{x}} v(\mathrm{x}, t)-\frac{\lambda^{2}}{2}|\mathrm{x}|^{2} v(\mathrm{x}, t), \quad(\mathrm{x}, t) \in \mathbb{R}^{N} \times[0, T), \\
v(\mathrm{x}, T) & =\psi_{\mathrm{m}, T, \lambda}(\mathrm{x})=e^{\frac{1}{2}\left(\sum_{j=1}^{N} m_{j}+\frac{N}{2}\right) \lambda T} \otimes_{j=1}^{N} h_{m_{j}, \lambda}(\mathrm{x}), \quad \mathrm{x} \in \mathbb{R}^{N},
\end{aligned}
$$

namely, one such pair for each $m_{j} \in \mathbb{N}$ and every $j$, where the $h_{m_{j}, \lambda}$ 's are the Hermite functions of Lemma 1. Accordingly, this means that we are considering as many pairs of such systems as is necessary to take into account the whole pure point spectrum of the elliptic operator on the right-hand side. We remark that (70)-(71) constitutes a generalization of (31)-(32), the latter system being associated with the bottom of the spectrum where $m_{j}=0$ for each $j$. Whereas the associated measures remain suitably normalized, the drawback is that they are no longer positive according to the following result:

Lemma 2. Let us write $\mathrm{m}:=\left(m_{1}, \ldots, m_{N}\right) \in \mathbb{N}^{N}$ and let us consider the sequence of measures

$$
\mu_{\mathrm{m}, \lambda}(F)=\int_{F} d \mu_{\mathrm{m}, \lambda}(\mathrm{x}, \mathrm{y})
$$

where

$$
\mu_{\mathrm{m}, \lambda}(\mathrm{x}, \mathrm{y})=\varphi_{\mathrm{m}, 0, \lambda}(\mathrm{x}) \psi_{\mathrm{m}, T, \lambda}(\mathrm{y}) g_{\lambda}(\mathrm{x}, T, \mathrm{y})
$$

and $F \in \mathcal{B}_{N} \times \mathcal{B}_{N}$. Then we have

$$
\int_{\mathbb{R}^{N} \times \mathbb{R}^{N}} d \mu_{\mathrm{m}, \lambda}(\mathrm{x}, \mathrm{y})=1
$$

and for every $\mathrm{m} \neq 0$ the $\mu_{\mathrm{m}, \lambda}$ 's are signed measures.

Proof. According to Proposition A.1 of the appendix we have

$$
=\sum_{\mathrm{n} \in \mathbb{N}^{N}} e^{-\left(\sum_{j=1}^{N} n_{j}+\frac{N}{2}\right) \lambda T} \otimes_{j=1}^{N} h_{n_{j}, \lambda}(\mathrm{x}) \times \otimes_{j=1}^{N} h_{n_{j}, \lambda}(\mathrm{y})
$$

for Mehler's kernel (38), where the series converges absolutely and uniformly for all $\mathrm{x}, \mathrm{y} \in \mathbb{R}^{N}$. Moreover, since the tensor products $\otimes_{j=1}^{N} h_{m_{j}, \lambda}$ provide an orthonormal basis of $L^{2}\left(\mathbb{R}^{N}, \mathbb{C}\right)$ we have

$$
\int_{\mathbb{R}^{N}} d \mathrm{x} \otimes_{j=1}^{N} h_{m_{j}, \lambda}(\mathrm{x}) \times \otimes_{j=1}^{N} h_{n_{j}, \lambda}(\mathrm{x})=\prod_{j=1}^{N} \delta_{m_{j}, n_{j}}
$$

for all $m_{j}, n_{j} \in \mathbb{N}$. Consequently, substituting $\varphi_{\mathrm{m}, 0, \lambda}, \psi_{\mathrm{m}, T, \lambda}$ and (75) into the left-hand side of (74) and taking (76) into account we obtain

$$
\begin{aligned}
& \int_{\mathbb{R}^{N} \times \mathbb{R}^{N}} d \mathrm{x} d \mathrm{y} \varphi_{\mathrm{m}, 0, \lambda}(\mathrm{x}) \psi_{\mathrm{m}, T, \lambda}(\mathrm{y}) g_{\lambda}(\mathrm{x}, T, \mathrm{y}) \\
= & \sum_{\mathrm{n} \in \mathbb{N}^{N}} e^{\sum_{j=1}^{N}\left(m_{j}-n_{j}\right) \lambda T} \prod_{j=1}^{N} \delta_{m_{j}, n_{j}}=1 .
\end{aligned}
$$


Finally, the $\mu_{\mathrm{m}, \lambda}$ 's are signed measures because of the existence of real zeroes for each $h_{m_{j}, \lambda}$ when $m_{j} \neq 0$.

The fact that the above measures are signed prevents one from applying directly the general results of Section 2 to (70)-(71) with a fixed $m \neq 0$, as it would prevent one from applying the main result of [2] briefly discussed at the end of Section 2. However, we can still save the day by constructing a oneparameter family of bona fide probability measures from all the $\mu_{\mathrm{m}, \lambda}$ 's. Indeed the following result is valid, where we call a measure non-Markovian whenever (22) does not hold:

Lemma 3. For each $\lambda>0$ there exists a one-parameter family $\left(\hat{\mu}_{\lambda, \theta}\right)_{\theta>0}$ of positive, non-Markovian measures of the form

$$
\hat{\mu}_{\lambda, \theta}(F)=\int_{F} d \hat{\mu}_{\lambda, \theta}(\mathrm{x}, \mathrm{y})
$$

where $F \in \mathcal{B}_{N} \times \mathcal{B}_{N}$, which satisfy

$$
\int_{\mathbb{R}^{N} \times \mathbb{R}^{N}} d \hat{\mu}_{\lambda, \theta}(\mathrm{x}, \mathrm{y})=1
$$

and which disintegrate into a statistical mixture of the $\mu_{\mathrm{m}, \lambda}$ 's. In other words, for each $\lambda>0$, each $\theta>0$ and every $\mathrm{m} \in \mathbb{N}^{N}$ there exist numbers $p_{\mathrm{m}, \lambda, \theta}>0$ such that

$$
\hat{\mu}_{\lambda, \theta}=\sum_{\mathbf{m} \in \mathbb{N}^{N}} p_{\mathbf{m}, \lambda, \theta} \mu_{\mathbf{m}, \lambda} \text { where } \sum_{\mathbf{m} \in \mathbb{N}^{N}} p_{\mathbf{m}, \lambda, \theta}=1 .
$$

In fact, it is sufficient to take

$$
\hat{\mu}_{\lambda, \theta}(\mathrm{x}, \mathrm{y})=(2(\cosh (\lambda(\theta+1) T)-1))^{\frac{N}{2}} g_{\lambda}(\mathrm{x}, T, \mathrm{y}) g_{\lambda}(\mathrm{x}, \theta T, \mathrm{y}) .
$$

Proof. From the series expansion (75) and the orthogonality relations (76) we have

$$
\begin{aligned}
& \int_{\mathbb{R}^{N} \times \mathbb{R}^{N}} d \times d y g_{\lambda}(\mathrm{x}, T, \mathrm{y}) g_{\lambda}(\mathrm{x}, \theta T, \mathrm{y}) \\
= & \sum_{\mathbf{m} \in \mathbb{N}^{N}} e^{-\left(\sum_{j=1}^{N} m_{j}+\frac{N}{2}\right) \lambda(\theta+1) T} \\
= & \left(2 \sinh \left(\lambda(\theta+1) \frac{T}{2}\right)\right)^{-N}
\end{aligned}
$$

by summing the underlying geometric series, from which we obtain (77) as a consequence of (78) and the identity

$$
4 \sinh ^{2}\left(\lambda(\theta+1) \frac{T}{2}\right)=2(\cosh (\lambda(\theta+1) T)-1) .
$$


As for the second statement of the lemma, let us define the sequence of numbers

$$
p_{\mathrm{m}, \lambda, \theta}:=(2(\cosh (\lambda(\theta+1) T)-1))^{\frac{N}{2}} e^{-\left(\sum_{j=1}^{N} m_{j}+\frac{N}{2}\right) \lambda(\theta+1) T}>0 .
$$

Summing as above we get

$$
\sum_{\mathbf{m} \in \mathbb{N}^{N}} p_{\mathrm{m}, \lambda, \theta}=1
$$

as required. Therefore, taking into account (73), (75) and (79) we obtain

$$
\begin{aligned}
& \sum_{\mathbf{m} \in \mathbb{N}^{N}} p_{\mathbf{m}, \lambda, \theta} \mu_{\mathrm{m}, \lambda}(\mathrm{x}, \mathrm{y}) \\
= & g_{\lambda}(\mathrm{x}, T, \mathrm{y}) \sum_{\mathbf{m} \in \mathbb{N}^{N}} p_{\mathrm{m}, \lambda, \theta} \varphi_{\mathrm{m}, 0, \lambda}(\mathrm{x}) \psi_{\mathrm{m}, T, \lambda}(\mathrm{y}) \\
= & (2(\cosh (\lambda(\theta+1) T)-1))^{\frac{N}{2}} g_{\lambda}(\mathrm{x}, T, \mathrm{y}) \\
& \times \sum_{\mathbf{m} \in \mathbb{N}^{N}} e^{-\left(\sum_{j=1}^{N} m_{j}+\frac{N}{2}\right) \lambda \theta T} \otimes_{j=1}^{N} h_{m_{j}, \lambda}(\mathrm{x}) \times \otimes_{j=1}^{N} h_{m_{j}, \lambda}(\mathrm{y}) \\
= & \hat{\mu}_{\lambda, \theta}(\mathrm{x}, \mathrm{y})
\end{aligned}
$$

according to (78), which is the desired conclusion.

REMARK. Strictly speaking, the measure $\hat{\mu}_{\lambda, \theta}$ does not exist for $\theta=0$ but the limit

$$
\hat{\mu}_{\lambda,+}(\mathrm{x}, \mathrm{y}):=\lim _{\theta \rightarrow 0_{+}} \hat{\mu}_{\lambda, \theta}(\mathrm{x}, \mathrm{y})=(2(\cosh (\lambda T)-1))^{\frac{N}{2}} g_{\lambda}(\mathrm{x}, T, \mathrm{y}) \delta(\mathrm{x}-\mathrm{y})
$$

does, by virtue of the fact that $g_{\lambda}$ is Green's function associated with the partial differential equation in (31). Said differently, Lemma 3 and its proof remain valid for the measure $\hat{\mu}_{\lambda,+}$ associated with (80) since we have

$$
\sum_{\mathbf{m} \in \mathbb{N}^{N}} \otimes_{j=1}^{N} h_{m_{j}, \lambda}(\mathrm{x}) \times \otimes_{j=1}^{N} h_{m_{j}, \lambda}(\mathrm{y})=\delta(\mathrm{x}-\mathrm{y})
$$

in the sense of distributions for all $\mathrm{x}, \mathrm{y} \in \mathbb{R}^{N}$, as a consequence of the completeness of the $\otimes_{j=1}^{N} h_{m_{j}, \lambda}$ 's in $L^{2}\left(\mathbb{R}^{N}, \mathbb{C}\right)$.

The above developments now lead to the following result:

Proposition 4. For each $\lambda>0$, the Bernstein processes $Z_{\tau \in[0, T]}^{\lambda, \theta>0}$ associated with the above infinite hierarchy through the measures $\hat{\mu}_{\lambda, \theta}$ are stationary Gaussian and non-Markovian processes such that

$$
\mathbb{P}_{\mu}\left(Z_{t}^{\lambda, \theta} \in E\right)=\left(2 \pi \sigma_{\lambda, \theta}\right)^{-\frac{N}{2}} \int_{E} d x \exp \left[-\frac{|\mathrm{x}|^{2}}{2 \sigma_{\lambda, \theta}}\right]
$$


for each $t \in[0, T]$ and every $E \in \mathcal{B}_{N}$, where

$$
\sigma_{\lambda, \theta}=\frac{\sinh (\lambda(\theta+1) T)}{2 \lambda(\cosh (\lambda(\theta+1) T)-1)} .
$$

Furthermore, the components of $Z_{\tau \in[0, T]}^{\lambda, \theta}$ satisfy the relation

$$
\mathbb{E}_{\mu}\left(Z_{s}^{\lambda, \theta, i} Z_{t}^{\lambda, \theta, j}\right)=\frac{\cosh \left(\lambda\left(|t-s|-\frac{(\theta+1) T}{2}\right)\right)}{2 \lambda \sinh \left(\frac{\lambda(\theta+1) T}{2}\right)} \delta_{i, j}
$$

for all $s, t \in[0, T]$ and all $i, j \in\{1, \ldots, N\}$. Finally, the process $Z_{\tau \in[0, T]}^{\lambda,+}$ associated with the measure $\hat{\mu}_{\lambda,+}$ identifies in law with a stationary process whose components are all independent, one-dimensional and periodic Ornstein-Uhlenbeck processes.

Proof. The processes $Z_{\tau \in[0, T]}^{\lambda, \theta>0}$ are Gaussian and non-Markovian by virtue of (16) with Green's function (38) and the measures $\hat{\mu}_{\lambda, \theta}$. Furthermore, using the symmetry properties of $g_{\lambda}$ with respect to the spatial variables, and twice the related semi-group composition law (94) of the appendix, (17) with (78) becomes

$$
\begin{aligned}
& \mathbb{P}_{\mu}\left(Z_{t}^{\lambda, \theta} \in E\right) \\
= & (2(\cosh (\lambda(\theta+1) T)-1))^{\frac{N}{2}} \int_{E} d \times g_{\lambda}(\mathrm{x},(\theta+1) T, \mathrm{x})
\end{aligned}
$$

for every $t \in(0, T)$. The same result obtains for $t=0$ and $t=T$ as a consequence of (18)-(19), so that (81)-(82) follows immediately since

$$
\begin{aligned}
& g_{\lambda}(\mathrm{x},(\theta+1) T, \mathrm{x}) \\
= & \left(\frac{\lambda}{2 \pi \sinh (\lambda(\theta+1) T)}\right)^{\frac{N}{2}} \exp \left[-\frac{\lambda(\cosh (\lambda(\theta+1) T)-1)|\mathrm{x}|^{2}}{\sinh (\lambda(\theta+1) T)}\right] .
\end{aligned}
$$

We now turn to the proof of (83) by determining the Gaussian density of $\left(Z_{t_{1}}^{\lambda, \theta}, \ldots, Z_{t_{n}}^{\lambda, \theta}\right)$. Thus, using $\hat{\mu}_{\lambda, \theta}$ in (16) and integrating first over y and then over $x$ we may write

$$
\begin{aligned}
& \mathbb{P}_{\mu}\left(Z_{t_{1}}^{\lambda, \theta} \in E_{1}, \ldots, Z_{t_{n}}^{\lambda, \theta} \in E_{n}\right) \\
= & (2(\cosh (\lambda(\theta+1) T)-1))^{\frac{N}{2}} \int_{E_{1}} d \mathrm{x}_{1} \ldots \int_{E_{n}} d \mathrm{x}_{n} \\
& \times \prod_{k=2}^{n} g_{\lambda}\left(\mathrm{x}_{k}, t_{k}-t_{k-1}, \mathrm{x}_{k-1}\right) \times \int_{\mathbb{R}^{N}} d \times g_{\lambda}\left(\mathrm{x}_{1}, t_{1}, \mathrm{x}\right) g_{\lambda}\left(\mathrm{x},(\theta+1) T-t_{n}, \mathrm{x}_{n}\right) \\
= & (2(\cosh (\lambda(\theta+1) T)-1))^{\frac{N}{2}} \int_{E_{1}} d \mathrm{x}_{1} \ldots \int_{E_{n}} d \mathrm{x}_{n} \\
& \times \prod_{k=2}^{n} g_{\lambda}\left(\mathrm{x}_{k}, t_{k}-t_{k-1}, \mathrm{x}_{k-1}\right) \times g_{\lambda}\left(\mathrm{x}_{1},(\theta+1) T-\left(t_{n}-t_{1}\right), \mathrm{x}_{n}\right)
\end{aligned}
$$


where we have once again used the semi-group composition law (94) twice, so that the Gaussian density in $\mathbb{R}^{n N}$ reads

$$
\begin{aligned}
& (2(\cosh (\lambda(\theta+1) T)-1))^{\frac{N}{2}} \\
\times & \prod_{k=2}^{n} g_{\lambda}\left(x_{k}, t_{k}-t_{k-1}, x_{k-1}\right) \times g_{\lambda}\left(\mathrm{x}_{1},(\theta+1) T-\left(t_{n}-t_{1}\right), \mathrm{x}_{n}\right) \\
= & (2 \pi)^{-\frac{n N}{2}}\left(\frac{2 \lambda^{n}(\cosh (\lambda(\theta+1) T)-1)}{\sinh \left(\lambda\left((\theta+1) T-\left(t_{n}-t_{1}\right)\right)\right)}\right)^{\frac{N}{2}} \prod_{k=2}^{n}\left(\sinh \left(\lambda\left(t_{k}-t_{k-1}\right)\right)^{-\frac{N}{2}}\right. \\
\times & \exp \left[-\frac{\lambda}{2} \sum_{k=2}^{n} \frac{\cosh \left(\lambda\left(t_{k}-t_{k-1}\right)\right)\left(\left|\mathrm{x}_{k}\right|^{2}+\left|\mathrm{x}_{k-1}\right|^{2}\right)-2\left(\mathrm{x}_{k}, \mathrm{x}_{k-1}\right)_{\mathbb{R}^{N}}}{\sinh \left(\lambda\left(t_{k}-t_{k-1}\right)\right)}\right] \\
\times & \exp \left[-\frac{\lambda}{2} \frac{\cosh \left(\lambda\left((\theta+1) T-\left(t_{n}-t_{1}\right)\right)\left(\left|\mathrm{x}_{1}\right|^{2}+\left|\mathrm{x}_{n}\right|^{2}\right)-2\left(\mathrm{x}_{1}, \mathrm{x}_{n}\right)_{\mathbb{R}^{N}}\right.}{\sinh \left(\lambda\left((\theta+1) T-\left(t_{n}-t_{1}\right)\right)\right)}\right] .
\end{aligned}
$$

For the sake of clarity we now identify the corresponding matrix $C_{\lambda, \theta}^{-1}$ by considering the case $n=2$ separately from the case $n \geq 3$, as $C_{\lambda, \theta}^{-1}$ is no longer tridagonal. For $n=2$ we obtain

$$
C_{\lambda, \theta, k, k}^{-1}=\frac{\lambda \sinh (\lambda(\theta+1) T)}{\sinh \left(\lambda\left(t_{2}-t_{1}\right)\right) \sinh \left(\lambda\left((\theta+1) T-\left(t_{2}-t_{1}\right)\right)\right)} \quad \text { for } k=1,2
$$

and

$$
C_{\lambda, \theta, 2,1}^{-1}=C_{\lambda, \theta, 1,2}^{-1}=-\frac{\lambda}{\sinh \left(\lambda\left|t_{2}-t_{1}\right|\right)}-\frac{\lambda}{\sinh \left(\lambda\left((\theta+1) T-\left|t_{2}-t_{1}\right|\right)\right)},
$$

while for $n \geq 3$ we get

$$
\begin{gathered}
C_{\lambda, \theta, k, k}^{-1}= \begin{cases}\frac{\lambda \sinh \left(\lambda\left((\theta+1) T-\left(t_{n}-t_{2}\right)\right)\right)}{\sinh \left(\lambda\left(t_{2}-t_{1}\right)\right) \sinh \left(\lambda\left((\theta+1) T-\left(t_{n}-t_{1}\right)\right)\right)} \quad \text { for } k=1, \\
\frac{\lambda \sinh \left(\lambda\left(t_{k+1}-t_{k-1}\right)\right)}{\sinh \left(\lambda\left(t_{k+1}-t_{k}\right)\right) \sinh \left(\lambda\left(t_{k}-t_{k-1}\right)\right)} \quad \text { for } k=2, \ldots, n-1, \\
\frac{\lambda \sinh \left(\lambda\left((\theta+1) T-\left(t_{n-1}-t_{1}\right)\right)\right)}{\sinh \left(\lambda\left(t_{n}-t_{n-1}\right)\right) \sinh \left(\lambda\left((\theta+1) T-\left(t_{n}-t_{1}\right)\right)\right)} \quad \text { for } k=n,\end{cases} \\
C_{\lambda, \theta, k, k-1}^{-1}=C_{\lambda, \theta, k-1, k}^{-1}=-\frac{\lambda}{\sinh \left(\lambda\left|t_{k}-t_{k-1}\right|\right)} \quad \text { for } k=2, \ldots, n,
\end{gathered}
$$

and

$$
C_{\lambda, \theta, n, 1}^{-1}=C_{\lambda, \theta, 1, n}^{-1}=-\frac{\lambda}{\sinh \left(\lambda\left((\theta+1) T-\left|t_{n}-t_{1}\right|\right)\right)},
$$

all the remaining matrix elements being zero. In both cases we then obtain by inversion

$$
C_{\lambda, \theta, k, l}=\frac{\sinh \left(\lambda\left|t_{k}-t_{l}\right|\right)-\sinh \left(\lambda\left(\left|t_{k}-t_{l}\right|-(\theta+1) T\right)\right)}{2 \lambda(\cosh (\lambda(\theta+1) T)-1)}
$$


for all $k, l \in\{1, \ldots, n\}$ or, equivalently,

$$
C_{\lambda, \theta, k, l}=\frac{\cosh \left(\lambda\left(\left|t_{k}-t_{l}\right|-\frac{(\theta+1) T}{2}\right)\right)}{2 \lambda \sinh \left(\frac{\lambda(\theta+1) T}{2}\right)},
$$

so that (83) eventually holds. Let us now consider the case $\theta=0$, namely, the case corresponding to the measure $\hat{\mu}_{\lambda,+}$ defined from (80), for which (82) and (83) become

$$
\sigma_{\lambda}=\frac{\sinh (\lambda T)}{2 \lambda(\cosh (\lambda T)-1)}
$$

and

$$
\mathbb{E}_{\mu}\left(Z_{s}^{\lambda,+, i} Z_{t}^{\lambda,+, j}\right)=\frac{\cosh \left(\lambda\left(|t-s|-\frac{T}{2}\right)\right)}{2 \lambda \sinh \left(\frac{\lambda T}{2}\right)} \delta_{i, j}
$$

for all $i, j \in\{1, \ldots, N\}$, respectively. Let us also consider the forward Itô integral equation with periodic boundary conditions

$$
\begin{aligned}
& X_{t}=e^{-\lambda t} X_{0}+\int_{0}^{t} e^{-\lambda(t-\tau)} d W_{\tau}, \quad t \in[0, T], \\
& X_{0}=X_{T}
\end{aligned}
$$

rather than (44). It is known from Theorem 2.1 in [14] (see also [17] or Section 5 in [18] for the case $N=1$ ) that the solution to (86) can be written out explicitly and defines a non-Markovian centered Gaussian stationary process, namely, the so-called periodic Ornstein-Uhlenbeck process given by

$$
X_{t}=\frac{e^{-\lambda t}}{1-e^{-\lambda T}} \int_{0}^{T} e^{-\lambda(T-\tau)} d W_{\tau}+\int_{0}^{t} e^{-\lambda(t-\tau)} d W_{\tau}, \quad t \in[0, T],
$$

whose variance and covariance are given by (84) and (85), respectively. Therefore, $Z_{\tau \in[0, T]}^{\lambda,+}$ identifies in law with that process.

REMARKs. (1) It is of course also a posteriori clear that the processes of Proposition 4 are not Markovian since the covariances (83) do not factorize as the product of a function of $s$ times a function of $t$, in contrast to all the cases investigated in Section 3. Furthermore, a very different way of understanding such factorization properties in the Markovian case was put forward in Section 6 of [13], where the covariances were written as the product of two linearly independent solutions to some suitable Sturm-Liouville problems.

(2) Problem (86) is part of a more general class of linear stochastic differential equations that were investigated by several authors, including [14], [16]-[18] and some of the references therein. In this context we ought to mention an analysis of the law of the solution to (86) in one dimension carried out in Section 5 of [18], which establishes a relation with Bernstein processes whose state space is one-dimensional. The main tool used there is a formula of integration 
by parts proved directly on the underlying infinite-dimensional path space by means of Malliavin's calculus. This is in sharp contrast to the method we have developed in this section, as we have first constructed a one-parameter family of non-Markovian, $N$-dimensional Bernstein processes associated with the infinite system (70)-(71), which we have only a posteriori identified with the solution to $(86)$ when $\theta=0$.

(3) While the processes $Z_{\tau \in[0, T]}^{\lambda, \theta>0}$ materialize a one-parameter family of nonMarkovian random curves in $\mathbb{R}^{N}$, they might also occur naturally in completely different contexts, as the limiting process $Z_{\tau \in[0, T]}^{\lambda,+}$ does. Thus, this process is quite relevant to the mathematical investigation of certain quantum systems in equilibrium with a thermal bath since it identifies with the Gaussian process of mean zero used in Theorem 2.1 of [8] to compute the expectations of some relevant physical quantities in statistical mechanics. Indeed, their covariances coincide since the equality

$$
\frac{\cosh \left(\lambda\left(t-\frac{T}{2}\right)\right)}{2 \lambda \sinh \left(\frac{\lambda T}{2}\right)}=\frac{\exp [-\lambda t]+\exp [-\lambda(T-t)]}{2 \lambda(1-\exp [-\lambda T])}
$$

holds for every $t \in[0, T]$, the period there being the inverse temperature, and since the right-hand side of (87) is the preferred form to write the covariance in [8] and [12]. More generally, certain multidimensional time-periodic processes such as $Z_{\tau \in[0, T]}^{\lambda,+}$ have been useful to solve filtering, smoothing and prediction problems as in [14], and also have played an important rôle in areas as diverse as the analysis of random evolution of loops as in [16], or the elaboration of global shape models generated by periodic lattices as in Chapters 11 and 16 of [7], where boundaries of random planar solid objects were investigated. It is our contention that the whole family of processes $Z_{\tau \in[0, T]}^{\lambda, \theta>0}$ might have an important rôle to play in those areas as well.

(4) We have already noted that many of the above developments rest on the series expansion for Green's function which we prove in the appendix below. We can get expansions of the same kind for Green's functions associated with forward-backward systems of the form

$$
\begin{aligned}
\partial_{t} u(\mathrm{x}, t) & =\frac{1}{2} \Delta_{\mathrm{x}} u(\mathrm{x}, t)-\left(\frac{\lambda^{2}}{2}|\mathrm{x}|^{2}+\kappa W(\mathrm{x})\right) u(\mathrm{x}, t), \quad(\mathrm{x}, t) \in \mathbb{R}^{N} \times(0, T], \\
u(\mathrm{x}, 0) & =\varphi_{0}(\mathrm{x}), \quad \mathrm{x} \in \mathbb{R}^{N}
\end{aligned}
$$

and

$$
\begin{aligned}
-\partial_{t} v(\mathrm{x}, t) & =\frac{1}{2} \Delta_{\mathrm{x}} v(\mathrm{x}, t)-\left(\frac{\lambda^{2}}{2}|\mathrm{x}|^{2}+\kappa W(\mathrm{x})\right) v(\mathrm{x}, t), \quad(\mathrm{x}, t) \in \mathbb{R}^{N} \times[0, T), \\
v(\mathrm{x}, T) & =\psi_{T}(\mathrm{x}), \quad \mathrm{x} \in \mathbb{R}^{N},
\end{aligned}
$$

for suitable anharmonic potentials $W: \mathbb{R}^{N} \mapsto \mathbb{R}$ where $\kappa>0$. We defer the analysis of Bernstein processes associated with such systems to a separate publication. 
Acknowledgments. The research of the first author was supported in part by the FCT of the Portuguese government under Grants UID/MAT/04561/2013 and PTDC/MAT/120354/2010, as well as by funds from the group Probabilités et Statistiques of the Université de Lorraine. The research of the second author was also supported by the FCT under Grant PTDC/MAT/120354/2010. Last but not least, the first author also wishes to thank the GFMUL and the Complexo Interdisciplinar da Universidade de Lisboa for their warm hospitality.

We complete this article by proving an important property of (38) which we used in the main part of the article.

\section{Appendix: a series expansion for Mehler's N- dimensional kernel}

The notation in this appendix is, of course, the same as in the preceding sections. The expansion in question is the following:

Proposition A1. We have

$$
=\sum_{\mathrm{m} \in \mathbb{N}^{N}} e^{-\left(\sum_{j=1}^{N} m_{j}+\frac{N}{2}\right) \lambda t} \otimes_{j=1}^{N} h_{m_{j}, \lambda}(\mathrm{x}) \times \otimes_{j=1}^{N} h_{m_{j}, \lambda}(\mathrm{y})
$$

where the series converges absolutely for every $t \in(0, T]$ and uniformly for all $\mathrm{x}, \mathrm{y} \in \mathbb{R}^{N}$. Furthermore, (88) is indeed Green's function associated with the partial differential equations in (31).

Proof. We first prove the result for the one-dimensional case $N=1$, namely,

$$
\begin{aligned}
& \left(\frac{\lambda}{2 \pi \sinh (\lambda t)}\right)^{\frac{1}{2}} \exp \left[-\frac{\lambda\left(\cosh (\lambda t)\left(x^{2}+y^{2}\right)-2 x y\right)}{2 \sinh (\lambda t)}\right] \\
= & \sum_{m=0}^{+\infty} e^{-\left(m+\frac{1}{2}\right) \lambda t} h_{m, \lambda}(x) h_{m, \lambda}(y) .
\end{aligned}
$$

Our point of departure for this is Mehler's notable formula in the form

$$
\begin{aligned}
& \left(\frac{1}{\pi\left(1-\gamma^{2}\right)}\right)^{\frac{1}{2}} \exp \left[-\frac{\left(1+\gamma^{2}\right)\left(x^{2}+y^{2}\right)-4 \gamma x y}{2\left(1-\gamma^{2}\right)}\right] \\
= & \sum_{m=0}^{+\infty} \gamma^{m} h_{m}(x) h_{m}(y)
\end{aligned}
$$

valid for every $\gamma \in(-1,+1)$, which allows one to express the probability density of two jointly Gaussian variables as a power series in the correlation parameter 
$\gamma$ (see, e.g., [20]). By using the scaled Hermite functions (33) instead, we obtain

$$
\begin{aligned}
& \left(\frac{\lambda}{\pi\left(1-\gamma^{2}\right)}\right)^{\frac{1}{2}} \exp \left[-\frac{\lambda\left(\left(1+\gamma^{2}\right)\left(x^{2}+y^{2}\right)-4 \gamma x y\right)}{2\left(1-\gamma^{2}\right)}\right] \\
= & \sum_{m=0}^{+\infty} \gamma^{m} h_{m, \lambda}(x) h_{m, \lambda}(y),
\end{aligned}
$$

so that in order to prove (89) we only need to identify $\gamma$. To this end we first compare the argument of the exponential on the left-hand side of (89) with that of (90), which gives the two conditions

$$
\frac{1+\gamma^{2}}{1-\gamma^{2}}=\operatorname{coth}(\lambda t)
$$

and

$$
\frac{\gamma}{1-\gamma^{2}}=\frac{1}{2 \sinh (\lambda t)} .
$$

Dividing (91) by (92) then leads to the quadratic equation

$$
\gamma^{2}-2 \cosh (\lambda t) \gamma+1=0,
$$

whose only solution in the interval $(-1,+1)$ is

$$
\gamma=e^{-\lambda t} .
$$

The substitution of (93) into (90) then gives (89) after some simple algebraic manipulations. Now, using (89) we obtain

$$
\begin{aligned}
& \sum_{\mathbf{m} \in \mathbb{N}^{N}} e^{-\left(\sum_{j=1}^{N} m_{j}+\frac{N}{2}\right) \lambda t} \otimes_{j=1}^{N} h_{m_{j}, \lambda}(\mathrm{x}) \times \otimes_{j=1}^{N} h_{m_{j}, \lambda}(\mathrm{y}) \\
= & \prod_{j=1}^{N} \sum_{m_{j}=0}^{+\infty} e^{-\left(m_{j}+\frac{1}{2}\right) \lambda t} h_{m_{j}, \lambda}\left(x_{j}\right) h_{m_{j}, \lambda}\left(y_{j}\right) \\
= & \left(\frac{\lambda}{2 \pi \sinh (\lambda t)}\right)^{\frac{N}{2}} \prod_{j=1}^{N} \exp \left[-\frac{\lambda\left(\cosh (\lambda t)\left(x_{j}^{2}+y_{j}^{2}\right)-2 x_{j} y_{j}\right)}{2 \sinh (\lambda t)}\right]
\end{aligned}
$$

which is (88), and the uniform convergence of the series follows from the CrámerCharlier inequality which ensures the uniform boundedness of the $h_{m}$ 's in $m$ and in their argument (see, e.g., Section 10.18 in [6] and the references therein). Finally, using the series expansion (88) and the eigenvalue equation (36) of Lemma 1 we get

$$
\begin{aligned}
\partial_{t} g_{\lambda}(\mathrm{x}, t, \mathrm{y}) & =\frac{1}{2} \Delta_{\times} g_{\lambda}(\mathrm{x}, t, \mathrm{y})-\frac{\lambda^{2}|\mathrm{x}|^{2}}{2} g_{\lambda}(\mathrm{x}, t, \mathrm{y}), \\
(\mathrm{x}, t) & \in \mathbb{R}^{N} \times(0, T]
\end{aligned}
$$


for every $\mathrm{y} \in \mathbb{R}^{N}$, and

$$
\lim _{t \rightarrow 0_{+}} g_{\lambda}(\mathrm{x}, t, \mathrm{y})=\sum_{\mathbf{m} \in \mathbb{N}^{N}} \otimes_{j=1}^{N} h_{m_{j}, \lambda}(\mathrm{x}) \times \otimes_{j=1}^{N} h_{m_{j}, \lambda}(\mathrm{y})=\delta(\mathrm{x}-\mathrm{y})
$$

in the sense of distributions since the $\otimes_{j=1}^{N} h_{m_{j}, \lambda}$ 's constitute a complete orthonormal system in $L^{2}\left(\mathbb{R}^{N}, \mathbb{C}\right)$. Consequently, $g_{\lambda}$ is indeed Green's function associated with the partial differential equation in (31).

REMARK. Of course, the semi-group composition law

$$
\begin{aligned}
& g_{\lambda}(\mathrm{x}, s+t, \mathrm{y}) \\
= & \int_{\mathbb{R}^{N}} d \mathrm{z} g_{\lambda}(\mathrm{x}, s, \mathrm{z}) g_{\lambda}(\mathrm{z}, t, \mathrm{y})
\end{aligned}
$$

valid for all $\mathrm{x}, \mathrm{y} \in \mathbb{R}^{N}$ is inherent in the fact that $g_{\lambda}$ is Green's function for (31), but follows most directly from the series expansion (88) and the orthogonality relations

$$
\int_{\mathbb{R}^{N}} d \mathrm{x} \otimes_{j=1}^{N} h_{m_{j}, \lambda}(\mathrm{x}) \times \otimes_{j=1}^{N} h_{n_{j}, \lambda}(\mathrm{x})=\prod_{j=1}^{N} \delta_{m_{j}, n_{j}} .
$$

Furthermore, we also observe that (88) brings out the entire pure point spectrum of Lemma 1 in the argument of the exponential, a fact that is crucial in our construction of Section 4 .

\section{References}

[1] Albeverio, S., Yasue, K., Zambrini, J-C., Euclidean quantum mechanics: analytical approach, Annales de l'Institut Henri Poincaré, Physique Théorique, 49 (1989) 259-308.

[2] Beurling, A., An automorphism of product measures, Annals of Mathematics 72 189-200 (1960).

[3] Carlen, E. A., Conservative diffusions, Communications in Mathematical Physics 94 (1984) 293-315.

[4] Cruzeiro, A. B., Vuillermot, P-A., Forward-backward stochastic differential equations generated by Bernstein diffusions, Stochastic Analysis and Applications 33 (2015) 91-109.

[5] Cruzeiro, A. B., Zambrini, J-C., Malliavin calculus and Euclidean quantum mechanics, I. Functional calculus, Journal of Functional Analysis 96 (1991) 62-95.

[6] Erdélyi, A., Magnus, W., Oberhettinger, F., Tricomi, F. G., Higher Transcendental Functions, II, McGraw-Hill, Inc., New York (1953). 
[7] Grenander, U., General Pattern Theory. A Mathematical Study of Regular Structures, Oxford Mathematical Monographs, Clarendon Press, Oxford (1993).

[8] Høegh-Krohn, R, Relativistic quantum statistical mechanics in twodimensional space-time, Communications in Mathematical Physics $\mathbf{3 8}$ (1974) 195-224.

[9] Huang, Y., McColl, W. F., Analytical inversion of general tridiagonal matrices, Journal of Physics A: Mathematical and General 30 (1997) 79197933.

[10] Jamison, B., Reciprocal processes, Zeitschrift für Wahrscheinlichkeitstheorie und Verwandte Gebiete 30 (1974) 65-86.

[11] Karatzas, I., Shreve, S. E., Brownian Motion and Stochastic Calculus, Graduate Texts in Mathematics 113, Springer-Verlag, New York (1988).

[12] Klein, A., Landau, L. J., Periodic Gaussian Osterwalder-Schrader positive processes and the two-sided Markov property on the circle, Pacific Journal of Mathematics 94 (1981) 341-367.

[13] Kolsrud, T., Zambrini, J-C., An introduction to the classical limit of Euclidean quantum mechanics, Journal of Mathematical Physics 33 (1992) 1301-1334.

[14] Kwakernaak, H., Periodic linear differential stochastic processes, SIAM Journal on Control 13 (1975) 400-413.

[15] Messiah, A., Quantum Mechanics, Dover Books on Physics, Dover (2014).

[16] Norris, J. R., Ornstein-Uhlenbeck processes indexed by the circle, The Annals of Probability 26 (1998) 465-478.

[17] Pedersen, J., Periodic Ornstein-Uhlenbeck processes driven by Lévy processes, Journal of Applied Probability 39 (2002) 748-763.

[18] Roelly, S., Thieullen, M., A characterization of reciprocal processes via an integration by parts formula on the path space, Probability Theory and Related Fields 123 (2002) 97-120.

[19] Schwartz, L., Théorie des Distributions, Hermann, Paris (1973).

[20] Slepian, D., On the symmetrized Kronecker power of a matrix and extensions of Mehler's formula for Hermite polynomials, SIAM Journal on Mathematical Analysis 3 (1972) 606-616.

[21] Vuillermot, P-A., Zambrini, J-C., Bernstein diffusions for a class of linear parabolic partial differential equations, Journal of Theoretical Probability 27 (2014) 449-492. 